\title{
THE TRANSITION MATRIX METHOD FOR A 2D EDDY CURRENT INTERACTION PROBLEM
}

\author{
Lars Larsson $^{1}$ and Anders Rosell ${ }^{2}$ \\ ${ }^{1}$ Advanced NDT/ Department of Materials and Manufacturing Technology, \\ Chalmers University of Technology, SE-412 96 Göteborg, Sweden \\ ${ }^{2}$ Volvo Aero Corporation, SE-46181 Trollättan, Sweden
}

\begin{abstract}
A 2D model of the eddy current interaction problem that consists of an inhomogeneity in a conductive half space is presented. The applied analytical method of solution is the transition (T) matrix method. This involves use of the free space Green's function to generate a system of boundary integral relations. In this way, it is easy to identify the contributions to the total solution from each different scattering surface. The different parts are separated also in the computation of the impedance. This leads to low cost simulations in terms of computation time and qualify the method to be used to obtain probability of detection (POD) curves. The $\mathrm{T}$ matrix method is a building block method and the possibility to extend the geometry with several inhomogeneities and extra layers will be discussed. The model is compared with a Finite Element (FE) model and numerical examples for the case with a cylindrical inhomogeneity are given.
\end{abstract}

Keywords: T Matrix Method, Eddy Current, Nondestructive Testing

PACS: 81.70.Ex

\section{INTRODUCTION}

To obtain probability of detection (POD) curves by low cost simulations is very desirable. Today there are many fast computation methods that can be used to achieve that. The aim of this paper is to apply the transition ( $\mathrm{T}$ ) matrix method to a two dimensional eddy current interaction model. This method have successfully been used on a variety of scattering problems (see for example refs. [1], [2], [3]).

The model in the present paper consists of a finite scatterer, the defect on one side of an infinite surface and a source on the other side (see Fig. 1). The system consumes energy (the medium where the defect is located is lossy). The amount of energy it consumes is dependent of the input current in the coil and the impedance of the system. The position, size and shape of the defect affect this impedance. Therefore is it possible to detect and size the defect by measuring the impedance with the eddy current equipment. When the probe scans the surface and it approaches the position above the defect there is a change in measured impedance. In a simulation of the surface scan this difference in impedance needs to be calculated for several different positions

(C) 2012 American Institute of Physics 978-0-7354-1013-8/\$30.00 


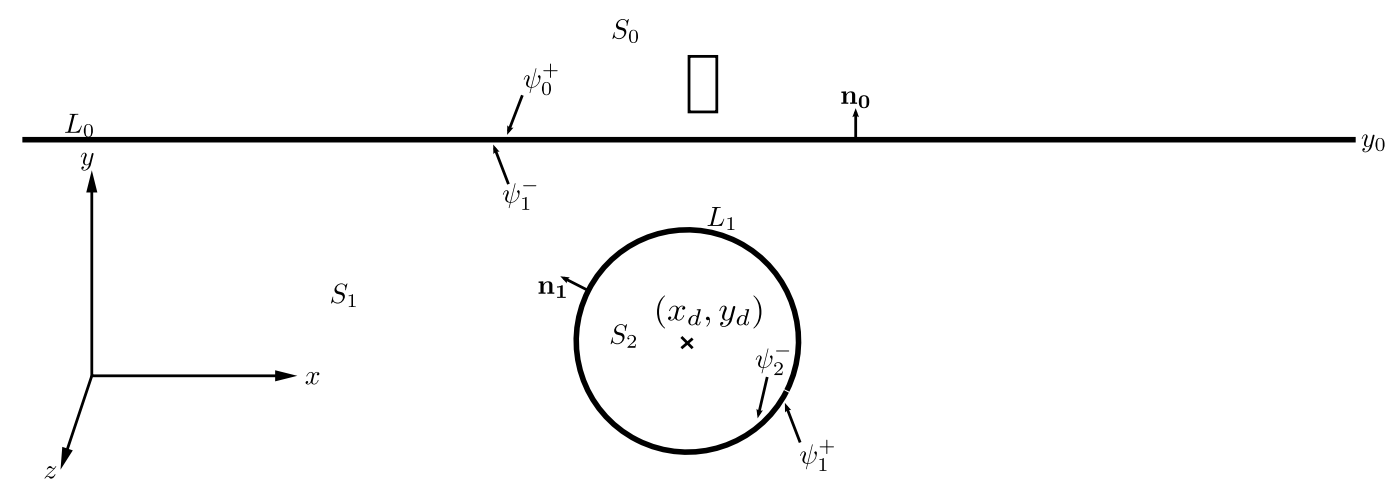

FIGURE 1. Geometry and notations.

of the probe. With the T matrix method a major part of the calculations does not have to be repeated when changing the position of the probe.

The analysis is based on use of the free space Green's function. Integration over the different homogeneous regions with standard Green's function technique gives a system of equations. To solve this system of equations, suitable expansions of the surface fields and the Green's function are needed. Plane wave basis functions and cylindrical wave basis functions is used for these expansions. The integrals over the defect will then give the $\mathrm{T}$ matrix that transforms the incoming field towards the defect to the outgoing field from the defect. The defect which represent a finite scatterer is completely characterized by its $\mathrm{T}$ matrix. The infinite scattering surface, on the other hand, will not be characterized by its $\mathrm{T}$ matrix but by its reflection and transmission coefficients.

There exist some references of $2 \mathrm{D}$ eddy current interaction models with a subsurface defect who use an analytical method of solution. The model of Hartfield and Bowler [4] of a deep subsurface crack and the model of Riaziat and Auld [5] of subsurface cracks in a layered medium are two examples, both using the Wiener-Hopf method of solution.

\section{INTEGRAL FORMULATION AND EXPANSIONS}

The scattering geometry is given in Fig. 1. All subregions $S_{i}, \quad i=0,1,2$, are assumed to be homogeneous, isotropic and linear. To simplify the calculations the surface $L_{0}$ is assumed to be a plane. The defect is assumed to be of cylindrical shape like a side drilled hole, which generates a diagonal $\mathrm{T}$ matrix. Other possible shapes are for example an elliptical cylinder or a strip (see [1]). The incoming field generated by the probe is assumed to be known. The $2 \mathrm{D}$ probe is an infinite conductor with current in the $z$-direction. This leads to two separate two dimensional problems for the field strength $\psi$. The first is $2 \mathrm{D}$ problem for the electric field strength $\psi=E_{z}$ or the magnetic vector potential $\psi=A_{z}$, and in the other a 2D problem for the magnetic field strength $\psi=H_{z}$. These problems are solved in the frequency domain with the time dependence $e^{-i \omega t}$. In this domain the field strengths satisfies the scalar Helmholtz equation

$$
\Delta \psi(\boldsymbol{r})+k^{2} \psi(\boldsymbol{r})=f,
$$

where $k^{2}=i \mu \omega \sigma+\omega^{2} \mu \varepsilon$ and $f$ is the known source term. Here $\mu$ is the magnetic permeability, $\varepsilon$ the electric permittivity and $\sigma$ the conductivity. Throughout this paper the same notations as in Karlsson [1] are mostly used. 
Now the free space Green's function $G\left(\boldsymbol{r}, \boldsymbol{r}^{\prime}, k\right)$ which satisfies

$$
\Delta G\left(\boldsymbol{r}, \boldsymbol{r}^{\prime}, k\right)+k^{2} G\left(\boldsymbol{r}, \boldsymbol{r}^{\prime}, k\right)=-\delta\left(\left|\boldsymbol{r}-\boldsymbol{r}^{\prime}\right|\right)
$$

is introduced. Then integration over the regions $S_{0}$ and $S_{1}$ and Green's theorem yields

$$
\begin{aligned}
-\int_{L_{0}}\left(G\left(\boldsymbol{r}, \boldsymbol{r}^{\prime}, k_{0}\right) \frac{\partial \psi_{0}^{+}(\boldsymbol{r})}{\partial n_{0}}-\psi_{0}^{+}(\boldsymbol{r}) \frac{\partial G\left(\boldsymbol{r}, \boldsymbol{r}^{\prime}, k_{0}\right)}{\partial n_{0}}\right) d L_{0}+\psi^{i n c}\left(\boldsymbol{r}^{\prime}\right) \\
= \begin{cases}\psi_{0}\left(\boldsymbol{r}^{\prime}\right), & \text { if } \boldsymbol{r}^{\prime} \in S_{0}, \\
0, & \text { if } \boldsymbol{r}^{\prime} \notin S_{0},\end{cases}
\end{aligned}
$$

where $\psi^{i n c}\left(r^{\prime}\right)=\int_{S_{0}} G\left(\boldsymbol{r}, \boldsymbol{r}^{\prime}, k_{0}\right) f d S_{0}$ is the incoming field and

$$
\begin{aligned}
& \int_{L_{0}}\left(G\left(\boldsymbol{r}, \boldsymbol{r}^{\prime}, k_{1}\right) \frac{\partial \psi_{1}^{-}(\boldsymbol{r})}{\partial n_{0}}-\psi_{1}^{-}(\boldsymbol{r}) \frac{\partial G\left(\boldsymbol{r}, \boldsymbol{r}^{\prime}, k_{1}\right)}{\partial n_{0}}\right) d L_{0} \\
& -\int_{L_{1}}\left(G\left(\boldsymbol{r}, \boldsymbol{r}^{\prime}, k_{1}\right) \frac{\partial \psi_{1}^{+}(\boldsymbol{r})}{\partial n_{1}}-\psi_{1}^{+}(\boldsymbol{r}) \frac{\partial G\left(\boldsymbol{r}, \boldsymbol{r}^{\prime}, k_{1}\right)}{\partial n_{1}}\right) d L_{1}= \begin{cases}\psi_{1}\left(\boldsymbol{r}^{\prime}\right), & \text { if } \boldsymbol{r}^{\prime} \in S_{1}, \\
0, & \text { if } \boldsymbol{r}^{\prime} \notin S_{1} .\end{cases}
\end{aligned}
$$

Here $\psi^{ \pm}$is the surface field on the positive $(+)$or negative $(-)$side of the boundary with respect to its normal vector $\hat{\boldsymbol{n}}$. As mentioned above this system of equations is solved by expanding the Green's function and the surface fields in suitable basis functions. The plane wave basis functions are defined as

$$
\varphi(\boldsymbol{k}, \boldsymbol{r})=\frac{1}{\sqrt{8 \pi}} e^{i \boldsymbol{k} \cdot \boldsymbol{r}}
$$

where the vector $\boldsymbol{k}$ is defined as $\boldsymbol{k}=(q, h)=k(\cos (\alpha), \sin (\alpha))$ and $\boldsymbol{r}=r(\cos (\phi), \sin (\phi))$. Here the symbols $(q, h)$ for the vector components are introduced to further reduce the length of the expressions. The definition of the cylindrical wave basis functions are

$$
\begin{aligned}
& R e \chi_{n}(\boldsymbol{r}, k)=\frac{\sqrt{\epsilon_{m}}}{2} J_{m}(k r) \begin{cases}\cos (m \phi), & \text { if } \varsigma=e, \\
\sin (m \phi), & \text { if } \varsigma=o,\end{cases} \\
& \chi_{n}(\boldsymbol{r}, k)=\frac{\sqrt{\epsilon_{m}}}{2} H_{m}^{(1)}(k r) \begin{cases}\cos (m \phi), & \text { if } \varsigma=e, \\
\sin (m \phi), & \text { if } \varsigma=o,\end{cases}
\end{aligned}
$$

where $\epsilon_{n}=2-\delta_{n, 0}, J_{m}$ is a Bessel function and $H_{m}^{(1)}$ is a Hankel function of the first kind. Here the multi-index $n=m \varsigma$ where $m \in \mathbf{N}, \varsigma=o$ denotes odd and $\varsigma=e$ denotes even has been introduced.

The surface field on $L_{0}$ is convenient to expand in plane waves

$$
\begin{gathered}
\psi_{0}^{+}(\boldsymbol{r})=\int_{C_{+}} \beta(\alpha) \varphi\left(\boldsymbol{k}_{\mathbf{0}}, \boldsymbol{r}\right) d \alpha \\
\frac{\partial \psi_{0}^{+}(\boldsymbol{r})}{\partial n}=\int_{C_{+}} \gamma(\alpha) \frac{\partial \varphi\left(\boldsymbol{k}_{\mathbf{0}}, \boldsymbol{r}\right)}{\partial n} d \alpha,
\end{gathered}
$$

where $\beta$ and $\gamma$ are the unknown expansion coefficients. The complex integration contours are chosen such that the set of plane waves $\varphi(\boldsymbol{k}, \boldsymbol{r})$ forms a complete set of basis functions. $C_{+}$is a contour from $\alpha=\pi / 4+i \infty$ to $3 \pi / 4-i \infty$ subject to 
$\tan (\Re(\alpha))=\operatorname{coth}(\Im(\alpha))$. The $C_{-}$contour which also will be used is defined as $\alpha+\pi \in C_{-}$if $\alpha \in C_{+}$. On $C_{+} q \in \mathbf{R}$ and $\operatorname{Im}(h) \geq 0$ and on $C_{-} q \in \mathbf{R}$ and $\operatorname{Im}(h) \leq 0$. Thus $q$ is a Fourier transform variable in the $x$ direction. Note that since $\operatorname{Im}(h) \geq 0$ on $C_{+}$and $\operatorname{Im}(h) \leq 0$ on $C_{-} e^{i h y}$ will be bounded if $y \geq 0$ or if $y \leq 0$. The surface field on $L_{1}$ is convenient to expand in cylindrical waves

$$
\begin{aligned}
\psi_{2}^{-} & =\sum_{n} \zeta_{n} \operatorname{Re} \chi_{n}\left(\boldsymbol{r}, k_{2}\right), \\
\frac{\partial \psi_{2}^{-}}{\partial n} & =\sum_{n} \zeta_{n} \frac{\partial \operatorname{Re} \chi_{n}\left(\boldsymbol{r}, k_{2}\right)}{\partial n},
\end{aligned}
$$

where $\zeta_{n}$ are unknown expansion coefficients. Note that the derivative of the surface field on $L_{1}$ is equal to the derivative of the expansion, but that is not the case on $L_{0}$. The reason is that the expansion on $L_{1}$ is valid everywhere in $S_{2}$ while the expansion on $L_{0}$ exists only on $L_{0}[3]$.

Expansions of the Green's function are given in [6], where necessary transformations between different basis functions also can be found. The plane wave expansion is

$$
G\left(\boldsymbol{r}, \boldsymbol{r}^{\prime}, k\right)=2 i \int_{C_{ \pm}} \varphi\left(\boldsymbol{k}, \boldsymbol{r}^{\prime}\right) \varphi(-\boldsymbol{k}, \boldsymbol{r}) d \alpha
$$

with integration over the $C_{+}$contour if $y^{\prime}>y$ and over $C_{-}$if $y^{\prime}<y$ (i.e. waves traveling in the direction from $y$ towards $\left.y^{\prime}\right)$. The expansion of $G\left(\boldsymbol{r}, \boldsymbol{r}^{\prime}, k\right)$ in cylindrical waves is

$$
G\left(\boldsymbol{r}, \boldsymbol{r}^{\prime}, k\right)=i \sum_{n} \operatorname{Re} \chi_{n}\left(r_{<}, k\right) \chi_{n}\left(r_{>}, k\right)
$$

where $\boldsymbol{r}_{<}$is the smaller and $\boldsymbol{r}_{>}$the greater of $r^{\prime}$ and $r$.

The next step is to introduce the boundary conditions $\psi_{i}^{+}=\psi_{j}^{-}$and $\frac{\partial \psi_{i}^{+}}{\partial n}=b_{i j} \frac{\partial \psi_{j}^{-}}{\partial n}$ where $b_{i j}=\frac{\mu_{i}}{\mu_{j}}$ if $\psi=E_{z}$ or $\psi=A_{z}$ and $b_{i j}=\frac{\varepsilon_{i}}{\varepsilon_{j}}$ if $\psi=H_{z}$. Then it is possible to express equation (3) and (4) in terms of the given expansions. To solve the system of equations it is also needed to make transformations between the cylindrical wave functions and the plane wave functions

$$
\begin{gathered}
\varphi(\boldsymbol{k}, \boldsymbol{r})=\sum_{n} D_{n}^{\dagger}(\alpha) \operatorname{Re} \chi_{n}(\boldsymbol{r}, k), \\
\chi_{n}(\boldsymbol{r}, k)=2 \int_{C_{ \pm}} D_{n}(\alpha) \varphi(\boldsymbol{k}, \boldsymbol{r}) d \alpha,
\end{gathered}
$$

where the integration is over $C_{+}$if $y>0$ and over $C_{-}$if $y<0$ (i.e. upwards traveling plane waves above the origin of the cylindrical wave and downwards traveling plane waves below the origin of the cylindrical wave). Here the transformation coefficients $D_{n}(\alpha)$ are defined as

$$
D_{n}(\alpha)=i^{-m}\left(\frac{\epsilon_{m}}{2 \pi}\right)^{\frac{1}{2}} \begin{cases}\cos (m \alpha), & \text { if } \varsigma=e \\ \sin (m \alpha), & \text { if } \varsigma=o\end{cases}
$$

and $D_{n}^{\dagger}$ in the same way except for a change of sign in the exponential $\left(i^{m}\right)$. 


\section{THE T MATRIX SOLUTION}

In this section the expansions above is used to solve the integral formulation of Helmholtz equation ((3) and (4)) for our geometry. Begin with equation (3) with $\boldsymbol{r}^{\prime} \notin S_{0}$ and the Green's function expanded in plane waves (eq. (12)). Then using the expansions (8) and (9), and changing the order of integration, yields the incoming field as an expansion in downwards traveling plane waves

$$
\psi_{i n c}\left(\boldsymbol{r}^{\prime}\right)=\int_{C_{-}} a_{i n c}(\alpha) \varphi\left(\boldsymbol{k}_{\mathbf{0}}, \boldsymbol{r}^{\prime}\right) d \alpha
$$

where

$$
a_{i n c}(\alpha)=\int_{C_{+}}-\beta\left(\alpha^{\prime}\right) 2 i Q\left(-\boldsymbol{k}_{\mathbf{0}}, \boldsymbol{k}_{\mathbf{0}}^{\prime}\right) d \alpha^{\prime}+\int_{C_{+}} \gamma\left(\alpha^{\prime}\right) 2 i Q\left(\boldsymbol{k}_{\mathbf{0}}^{\prime},-\boldsymbol{k}_{\mathbf{0}}\right) d \alpha^{\prime}, \quad \alpha \in C_{-} .
$$

Here

$$
Q\left(\boldsymbol{k}_{\boldsymbol{i}}, \boldsymbol{k}_{\boldsymbol{j}}\right)=\int_{L_{0}} \frac{\partial \varphi\left(\boldsymbol{k}_{\boldsymbol{i}}, \boldsymbol{r}\right)}{\partial n} \varphi\left(\boldsymbol{k}_{\boldsymbol{j}}, \boldsymbol{r}\right) d L_{0} .
$$

In our coordinates the integration over $L_{0}$ is an integration over $x$ and the $n$-derivative is the $y$-derivative. Then use of the identity $2 \pi \delta(\xi)=\int_{-\infty}^{\infty} e^{-i \xi x} d x$ yields $Q\left(\boldsymbol{k}_{\boldsymbol{i}}, \boldsymbol{k}_{\boldsymbol{j}}\right)=$ $i h_{i} \varphi\left(h_{i}, y_{0}\right) \varphi\left(h_{j}, y_{0}\right) \sqrt{\pi / 2} \delta\left(q_{i}+q_{j}\right)$. Now the integration over the $C_{+}$contour in equation (18) becomes very simple.

Now turn to equation (4). When $\boldsymbol{r}^{\prime} \in S_{0}$, the Green's function expansion (eq. (12)) with integration over the $C_{+}$contour can be used. This gives an integrand containing plane waves when integration is over the circular boundary $L_{1}$ of the cylindrical defect. To avoid that, these plane waves are transformed to cylindrical waves using transformation (14) which results in

$$
\begin{aligned}
& \int_{C_{+}}\left(\beta\left(\alpha^{\prime}\right) Q\left(-\boldsymbol{k}_{\mathbf{1}}, \boldsymbol{k}_{\mathbf{0}}^{\prime}\right)-\gamma\left(\alpha^{\prime}\right) b_{10} Q\left(\boldsymbol{k}_{\mathbf{0}}^{\prime},-\boldsymbol{k}_{\mathbf{1}}\right)\right) d \alpha^{\prime} \\
&=\sum_{n^{\prime} m} D_{n^{\prime}}^{\dagger}(\alpha+\pi) \operatorname{Re} Q_{n^{\prime} m} \zeta_{m}, \quad \alpha \in C_{+}
\end{aligned}
$$

where

$$
R e Q_{n^{\prime} m}=\int_{L_{1}}\left(\frac{\partial R e \chi_{n^{\prime}}\left(\boldsymbol{r}, k_{1}\right)}{\partial n} R e \chi_{m}\left(\boldsymbol{r}, k_{2}\right)-R e \chi_{n^{\prime}}\left(\boldsymbol{r}, k_{1}\right) b_{12} \frac{\partial R e \chi_{m}\left(\boldsymbol{r}, k_{2}\right)}{\partial n}\right) d L_{1} .
$$

The integration over $L_{1}$ is in polar coordinates and results in a diagonal matrix. If the same equation (4) is used, but with $\boldsymbol{r}^{\prime} \in S_{2}$ then the Green's function can be expanded in cylindrical waves according to (13). To avoid cylindrical waves when integrating over $L_{0}$ transformation (15) is used to transform them into plane waves and get

$$
\sum_{m} Q_{n m} \zeta_{m}=2 \int_{C_{+}} D_{n}(\alpha) \int_{C_{+}}\left(\beta\left(\alpha^{\prime}\right) Q\left(\boldsymbol{k}_{\mathbf{1}}, \boldsymbol{k}_{\mathbf{0}}^{\prime}\right)-\gamma\left(\alpha^{\prime}\right) b_{10} Q\left(\boldsymbol{k}_{\mathbf{0}}^{\prime}, \boldsymbol{k}_{\mathbf{1}}\right)\right) d \alpha^{\prime} d \alpha, \quad \forall n
$$

where

$$
Q_{n m}=\int_{L_{1}}\left(\frac{\partial \chi_{n}\left(\boldsymbol{r}, k_{1}\right)}{\partial n} R e \chi_{m}\left(\boldsymbol{r}, k_{2}\right)-\chi_{n}\left(\boldsymbol{r}, k_{1}\right) b_{12} \frac{\partial R e \chi_{m}\left(\boldsymbol{r}, k_{2}\right)}{\partial n}\right) d L_{1} .
$$


Now the equations ((18), (20) and (22)) form a system of equations with three unknown expansion coefficients, $\beta(\alpha), \gamma(\alpha)$ and $\zeta_{m}$. The solution to these equations combined with the equations (3), (4) and (10), yields the field in any of the regions $S_{0}, S_{1}$ and $S_{2}$. It should be noted that equation (22) contains a double integration over the $C_{+}$ contour. One of these integrations has to be computed numerically. After elimination of $\beta(\alpha)$ and $\gamma(\alpha)$ the system is reduced to

$$
d_{m}=\sum_{n} A_{m n} c_{n}+2 c_{m}
$$

where

$$
A_{m n}=\sum_{n^{\prime}} T_{n^{\prime} n} \int_{C_{+}} 4 R_{10} D_{m}(\pi-\alpha) D_{n^{\prime}}^{\dagger}(\alpha+\pi) e^{2 i \sqrt{k_{1}^{2}-q_{1}^{2}} y_{0}} d \alpha
$$

and

$$
d_{m}=\int_{C_{+}} D_{m}(\pi-\alpha)\left(1-R_{10}\right) a_{i n c}\left(2 \pi-\arccos \left(\frac{q_{1}}{k_{0}}\right)\right) e^{i\left(\sqrt{k_{1}^{2}-q_{1}^{2}}-\sqrt{k_{0}^{2}-q_{1}^{2}}\right) y_{0}} d \alpha .
$$

Here the $\mathrm{T}$ matrix has been introduced as

$$
T_{n^{\prime} n}=\sum_{m} \operatorname{Re} Q_{n^{\prime} m} Q_{n m}^{-1}
$$

and $R_{i j}$ the reflection coefficient as

$$
R_{i j}=\frac{\sqrt{k_{i}^{2}-q_{i}^{2}}-b_{i j} \sqrt{k_{j}^{2}-q_{i}^{2}}}{\sqrt{k_{i}^{2}-q_{i}^{2}}+b_{i j} \sqrt{k_{j}^{2}-q_{i}^{2}}}
$$

where the square root is defined such that $\operatorname{Im} \sqrt{(*)} \geq 0$. The new unknown $c_{n}$ in equation (24) is defined as $c_{n}=\sum_{m} i Q_{n m} \zeta_{m}$. Then equation (24) is solved numerically to get the fields and calculate the impedance. Conditions for a solution to exist is given in [3] and further references given there. It should be noted that the matrix $A$ is independent of the incoming field.

\section{NUMERICAL CALCULATIONS}

In this section the analytical solution of the field is used to calculate the change of impedance due to a defect. This difference in impedance $\Delta Z=Z_{b}-Z_{a}$ can be calculated by integration over a surface enclosing the defect (see Auld and Moulder [7]). With the index $b$ denoting the case in presence of a defect and $a$ the case in absence of a defect $\Delta Z$ can be calculated as

$$
\Delta Z=\frac{1}{I^{2}} \int_{L_{\text {defect }}}\left(\boldsymbol{E}_{\boldsymbol{a}} \times \boldsymbol{H}_{\boldsymbol{b}}-\boldsymbol{E}_{\boldsymbol{b}} \times \boldsymbol{H}_{\boldsymbol{a}}\right) d L .
$$

On this surface the surface field expansion (10) can be used, thus this expression is convenient to use. By introducing the magnetic vector potential $A$, such that $B=\nabla \times A$ expression (29) can be written as

$$
\Delta Z=\frac{i \omega}{\mu I^{2}} \int_{L_{1}}\left(\psi_{a} \frac{\partial \psi_{b}}{\partial n}-\psi_{b} \frac{\partial \psi_{a}}{\partial n}\right) d L
$$



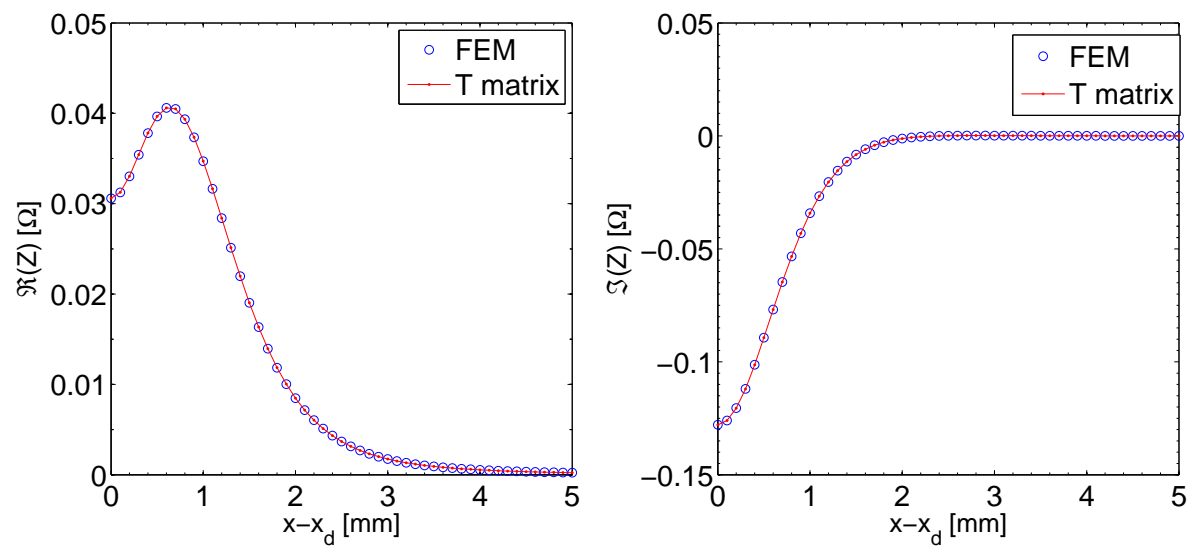

FIGURE 2. $\Delta Z$ as a function of distance from the defect.
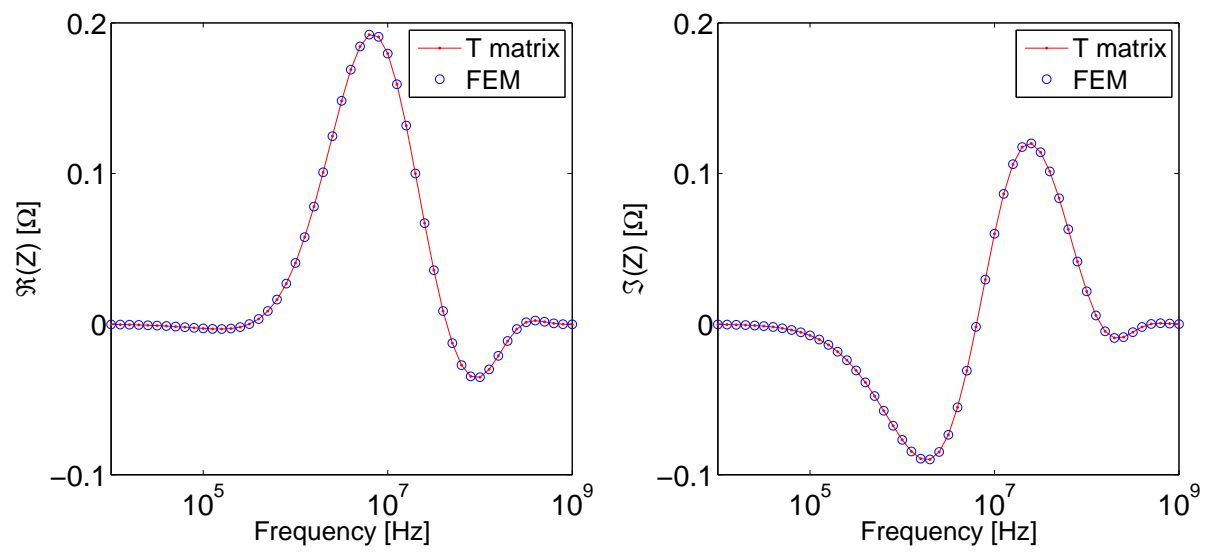

FIGURE 3. $\Delta Z$ as a function of frequency.

where $\psi=A_{z}$. Then the surface field expansion (10) is used to get

$$
\Delta Z=-\frac{i \omega}{\mu I^{2}} \sum_{m n} \zeta_{a n} \zeta_{b m} R e Q_{n m}
$$

Before using expression (31) the expansion coefficients of the incoming field has to be derived. With the input current $I$, the source term is $f=\mu_{0} J$, where $J$ is the input current density $J=I /$ conductor area. Then using the plane wave expansion of the Green's function and integration over the source yields the expansion coefficients of the incoming field

$$
\psi^{i n c}\left(r^{\prime}\right)=\int_{S} G\left(\boldsymbol{r}, \boldsymbol{r}^{\prime}, k_{0}\right) f d S=\int_{C_{-}} \underbrace{\int_{S} \varphi\left(-\boldsymbol{k}_{\mathbf{0}}, \boldsymbol{r}\right) \mu_{0} J d S}_{a_{i n c}(\alpha)} \varphi\left(\boldsymbol{k}_{\mathbf{0}}, \boldsymbol{r}^{\prime}\right) d \alpha,
$$

where $S$ is the cross section of the conductor.

Now expression (31) is used to calculate $\Delta Z$ and compare the results with a finite element solution of the same problem. Both methods are implemented in MATLAB. Figure 2 shows $\Delta Z$ as a function of probe position and Fig. 3 shows $\Delta Z$ as a function of frequency. These numerical examples are just intended to serve as a comparison 
with the finite element method for realistic parameters. In both numerical examples the lift-off is $0.1 \mathrm{~mm}$ and the conductivity is $\sigma=0.58 \mathrm{MS} / \mathrm{m}$ which corresponds to the conductivity of titanium. The defect is positioned $0.1 \mathrm{~mm}$ below the surface and has a diameter of $1 \mathrm{~mm}$. The rectangular conductor is $1 \mathrm{~mm}$ high and $0.25 \mathrm{~mm}$ wide. In the surface scan simulation (Fig. 2) the input frequency is $1 \mathrm{MHz}$. Then the probe position is chosen close to the maximum amplitude $\left(x-x_{d}=0.6 \mathrm{~mm}\right)$ and the skin depth is varied by changing the frequency (Fig. 3). Both examples shows very good agreement of the solutions from the two methods.

\section{CONCLUDING REMARKS}

The T matrix method can be used to solve the eddy current interaction problem. The advantage compared to the finite element method is shorter computation times. The presented examples is solved 10 to 50 times faster with the $\mathrm{T}$ matrix method, depending on how many points that is used for the position of the probe. The difference in computation time increase with the increased amount of points. This comparisons are made on the same standard PC.

In the present paper the $2 \mathrm{D}$ problem with a cylindrical defect is treated, but it can also be extended to the 3D case. The 3D defect can be "sphere like" or an ellipsoid. An elliptical cylinder defect where one of the radii tends to zero can be used to model a strip-like crack. In similar manner it is possible to model disk-shaped defects from an ellipsoid. The subsurface defect can be positioned very near the surface, resulting in an imitation of an almost surface-breaking crack.

\section{ACKNOWLEDGEMENTS}

The authors wishes to thank Prof. Anders Boström and Dr. Peter Bövik for valuable discussions and for checking the derivations. The work in the present paper is a part of the European project "PICASSO" and the funding from the European Commission within the FP7 programme is gratefully acknowledged.

\section{REFERENCES}

1. A. Karlsson, J. Acoust. Soc. Am. 71(5), pp. 1083-1092 (1982).

2. G. Kristensson, J. Appl. Phys. 51(7), pp. 3486-3500 (1980).

3. G. Kristensson and S. Ström, J. Acoust. Soc. Am. 64(3), pp. 917-936 (1978).

4. N. Harfield and J. R. Bowler. R. B. Thompson, "Analytical calculations of eddy current probe impedance response to cracks" in Review of Progress in Quantitative Nondestructive Evaluation 13A, edited by D. O. Thompson and D. E. Chimenti, Plenum Press, New York, 1994, pp. 279-286.

5. M. Riaziat and B.A Auld, "Angular Spectrum Analysis applied to Undercladding Flaws and Dipole Probes" in Review of Progress in Quantitative Nondestructive Evaluation 3A, edited by D. O. Thompson and D. E. Chimenti, Plenum Press, New York, 1984, pp. 511-521.

6. A. Boström, G. Kristensson and S. Ström, "Transformation properties of plane, spherical and cylindrical scalar and vector wave functions" in Field Representations and Introduction to Scattering, volume 1 of Acoustic, electromagnetic and elastic wave scattering, edited by V.V Varadan, A. Lakhtakia and V.K Varadan, North-Holland, Amsterdam (1991).

7. B.A. Auld and J.C Moulder, J. Nondest. Eval. 18(1), pp. 3-36 (1999). 